\title{
DISEÑO DE ENSEÑANZA DE LAS PROPIEDADES GLOBALES DE LAS FUNCIONES A TRAVÉS DE SUS GRÁFICAS
}

\author{
Ortega, Tomás ${ }^{1}$ y Pecharromán, Cristina ${ }^{2}$ \\ ${ }^{1}$ Didáctica de la Matemática. Universidad de Valladolid \\ ${ }^{2}$ IES Valverde de Lucerna. Puebla de Sanabria (Zamora) \\ ortega@am.uva.es \\ cpecharromang@yahoo.es
}

\begin{abstract}
Resumen. En este artículo se presenta el diseño de enseñanza de las propiedades globales de las funciones a través de sus gráficas como respuesta a un problema de aprendizaje detectado en el aula. Se construye un diseño de enseñanza basado en la caracterización del proceso de aprendizaje que ofrece el marco teórico conocido como Enfoque Lógico Semiótico (ELOS), que pretende facilitar el aprendizaje de tales propiedades mediante la coordinación de los sistemas de representación.
\end{abstract}

Palabras clave. Diseño, enseñanza, propiedades, funciones, elos.

\section{Design of the teaching of global properties of functions through their graphs}

Summary. In this paper we represent the teaching design of the global properties of functions through their graphics as a response to a problem of learning detected in the classroom. We have built a teaching design based on the characterization of the process of learning which offers the theoretical frame known as Logic Semiotic Focus (ELOS), which aims to facilitate the learning of such properties by means of the coordination of representation systems.

Keywords. Design, teaching, properties, functions, elos.

\section{INTRODUCCIÓN}

En este artículo, se presentan algunas bases teóricas y de planificación que han orientado el desarrollo experimental de una investigación que está dirigida a la mejora del proceso de enseñanza-aprendizaje de las propiedades globales de las funciones (dominio, recorrido, monotonía, extremos, corte con ejes, signo, continuidad, tendencia, periodicidad, simetrías convexidad), en segundo ciclo de Educación Secundaria Obligatoria (ESO).

$\mathrm{Al}$ igual que se habla del estudio local de las funciones, basado en el análisis diferencial (Martínez 1969, pág. 381), consideramos el estudio global de las funciones a la interpretación de sus propiedades sin utilizar el cálculo diferencial. Por contra, este estudio se realiza utilizando las diferentes representaciones de las funciones: verbal, gráfica, numérica y algebraica, y es un contenido propio de la etapa educativa de ESO.
La investigación surge en el aula al observar las dificultades de los alumnos para interpretar la gráfica de una función no contextualizada y para expresar esta información en los diversos sistemas de representación, sobre todo en el numérico y en el algebraico, pues consideramos, y posteriormente utilizamos, el sistema verbal como un medio interpretativo de los conceptos en las citadas representaciones.

El currículo oficial y nuestra experiencia docente ponen de manifiesto la importancia de la interpretación de las propiedades globales de las funciones a través de sus gráficas, por su marcado carácter formativo e instrumental, por lo que se observa la conveniencia de analizar el proceso de enseñanza-aprendizaje de estos conceptos. Sin embargo, aunque el currículo señala la importancia de la contextualización de los contenidos matemáticos 
en ESO, consideramos que en $4^{\circ}$. ESO Opción B también es conveniente que el aprendizaje de estos conceptos se llegue a independizar del contexto real que le motiva, ya que puede haber interpretaciones correctas de un fenómeno real, sin que haya una reflexión adecuada del concepto matemático que se quiere enseñar. Se pretende conseguir el completo desarrollo cognitivo de los conceptos a través de los sistemas de representación, es decir, consolidar la interpretación de las propiedades globales de las funciones como conceptos matemáticos abstractos. Por tanto, esta investigación incide más en el carácter formativo del aprendizaje de estos conceptos que en su carácter funcional o instrumental.

Por otra parte, las ideas de diversos autores en investigación educativa han orientado el desarrollo de la investigación desde diversas perspectivas, entre ellas las que señalan la importancia del sistema de representación gráfico como medio para el aprendizaje de ciertos conceptos asociados a él.

En definitiva, es una investigación que parte del aula y pretende que sus resultados orienten la práctica educativa, lo que hace que la metodología cualitativa de investigación-acción haya sido la más adecuada para su desarrollo experimental, porque permite el estudio cualitativo de la práctica educativa y porque admite que el desarrollo de la investigación se adapte fácilmente a las condiciones materiales y humanas de cada momento.

El marco teórico ELOS (Socas 1997 y 2007) sienta las bases fundamentales del marco teórico que ha presidido la investigación. En este trabajo, se indica que el aprendizaje de los conceptos es un proceso de abstracción que está estructurado por unos estadios (semiótico, estructural y autónomo) perfectamente caracterizados. Considerando esta interpretación del proceso de aprendizaje, nosotros creemos que un proceso educativo que se desarrolle a través de dichos estadios facilitaría a los alumnos el aprendizaje significativo de los conceptos y su completo desarrollo cognitivo a través de los diversos sistemas de representación. Esta idea nos llevó a elaborar un diseño de enseñanza de las propiedades globales de las funciones estructurado por los estadios semiótico, estructural y autónomo, y donde unas acciones sobre las representaciones de los conceptos concretan el aprendizaje asociado a cada estadio.

Un análisis curricular de la Unidad Didáctica de Funciones y Gráficas en la etapa educativa de ESO permite un primer acercamiento a los conceptos y a sus representaciones. Dicho trabajo muestra el tratamiento didáctico que los textos hacen de las propiedades globales de las funciones, y también permite observar cuáles son los contenidos sobre los que se fundamenta el aprendizaje de estos conceptos. Por tanto, este estudio resulta ser un referente que posteriormente orienta la concreción de los aprendizajes asociados a cada estadio de desarrollo cognitivo.

Por último, tras la presentación de las características generales que presiden la elaboración del diseño de enseñanza, se muestra un ejemplo concreto y se describen algunas observaciones y conclusiones que se han derivado de la elaboración de este diseño de enseñanza.

\section{ANTECEDENTES DE LA INVESTIGACIÓN}

La búsqueda y estudio de antecedentes a esta investigación en bibliografía de Didáctica de la Matemática y en la base de datos MATHDI de ZDM permitieron apreciar ciertas líneas de pensamiento común en diversos autores, aunque cada investigación las hiciese propias de su autor. Estas ideas orientaron nuestro trabajo de investigación y, en concreto, han influido en el carácter y en la elaboración del diseño de enseñanza para el aprendizaje de las propiedades globales de las funciones.

Diversos autores -Cobo (1995), Bagni (2004), Dolores (2004), y Dolores y Cuevas (2007) - son favorables a desarrollar procesos de visualización para alcanzar diferentes objetivos dentro del proceso de aprendizaje de las matemáticas. Además, hay autores -Deulofeu (1995) y Bloch (2003) - que consideran que la representación gráfica es la más adecuada para iniciar el aprendizaje del concepto de función y sus propiedades. Estas ideas nos llevaron a plantear un diseño de enseñanza de las propiedades globales de las funciones a través de su representación gráfica, considerando que el punto de partida del aprendizaje debe ser el reconocimiento de los conceptos mediante la técnica perceptiva de la visualización.

Asimismo, se señala la importancia de la función semiótica del lenguaje gráfico -Bloch, 2003; Sanz-Lerma, 2004- y se destaca la contribución de este lenguaje al fundamento de otros aprendizajes y al desarrollo del conocimiento matemático -Bloch, 2003; Dolores, 2004-. Ambos aspectos contribuyen a reforzar nuestro interés por utilizar el sistema de representación gráfico como medio para iniciar el aprendizaje de las propiedades globales de las funciones.

Otro grupo de autores, entre ellos Azcárate (1997), Knuth (2000) y Bloch (2003), que se hace eco de las ideas de Schwarz y Dreyfus (1995), Duval (1993, 1998) y Bagni (2004), señalan la importancia del uso de las diferentes representaciones de los conceptos para su aprendizaje y la relevancia de conocer las relaciones entre las mismas. También se manifiesta la importancia de que el aprendizaje de los conceptos parta del conocimiento de los alumnos (Azcárate, 1995), o de sus intuiciones (Deulofeu, 1995). Estas consideraciones nos inducen a desarrollar una docencia que relacione los símbolos nuevos con los ya conocidos, destacando las relaciones entre las representaciones de los conceptos, posición que encaja perfectamente con el marco teórico ELOS.

Por último, varias investigaciones ponen de relieve algunas dificultades que podemos llegar a observar en el aprendizaje de estos conceptos. Destacamos la falta de coordinación de lo que ocurre gráficamente con las dos variables de la función, debida al escaso desarrollo del pensamiento covariacional (Dolores, 2004; Dolores y Cuevas, 2007) y la tendencia a centrar la atención en ciertos símbolos gráficos frente al estudio global de la gráfica, es decir, a discretizar la información que transmite la gráfica Leinhardt, Zaslavsky y Stein, 1990; Deulofeu, 1995; Dolores y Cuevas, 2007). 
Aunque nuestra investigación tiene una orientación diferente a la de los trabajos mencionados, sus ideas han encauzado la planificación de nuestro estudio, sobre todo en el planteamiento de la docencia de los conceptos, que es el tema que aquí nos ocupa, y se han hecho presentes en el desarrollo experimental de la investigación, donde se ha observado el valor de estas ideas.

\section{FUNDAMENTOS DEL MARCO TEÓRICO DE LA INVESTIGACIÓN}

Socas (1997) presenta un estudio en el que caracteriza las dificultades, los obstáculos y los errores en el aprendizaje de las matemáticas en Educación Secundaria Obligatoria, y lo ilustra desde la construcción del conocimiento algebraico. El autor clasifica las dificultades de aprendizaje en cinco grandes apartados, y considera que, en la práctica, estas dificultades se concretan en forma de obstáculos y se manifiestan en forma de errores. En concreto, el autor indica que los errores pueden tener origen en un obstáculo, en la ausencia de significado (motivada por la complejidad de los objetos y los procesos de pensamiento matemático) y en actitudes afectivas y emocionales.

En la investigación que desarrollamos, tienen especial relevancia las dificultades asociadas a la complejidad de los objetos matemáticos. Estas dificultades, por una parte, son debidas al uso del lenguaje habitual para favorecer la interpretación de los signos matemáticos, ya que el uso conjunto de ambos lenguajes puede originar conflictos de precisión, confusiones semánticas, conflictos de especificidad y de socialización; también considera dificultades asociadas a la pragmática y señala la influencia que tiene el contexto en la interpretación de significados. Por otra parte, considera que estas dificultades aparecen en el proceso de aprendizaje de los conceptos matemáticos, que el autor interpreta como un proceso de abstracción de los sistemas de representación de dichos conceptos, y donde distingue y caracteriza los estadios de desarrollo siguientes:

- Semiótico: Los signos nuevos adquieren significado y se definen a partir de los antiguos, ya conocidos. El sistema nuevo de signos es caracterizado por el sistema antiguo.

- Estructural: El sistema nuevo de símbolos se estructura según la organización del antiguo, y las dificultades para dar sentido a ciertos símbolos o propiedades se abordan considerando regularidades y patrones de comportamiento.

- Autónomo: El sistema nuevo adquiere significado en sí mismo, pero es una fuente de dificultades al encontrarnos con elementos que no pueden ser conocidos en términos del sistema antiguo. En estos casos se produce la generalización, proceso característico de las matemáticas en el desarrollo de sus signos.

Esta caracterización del aprendizaje de los objetos matemáticos, donde los aprendizajes que se alcanzan en cada estadio fundamentan los aprendizajes del estadio siguiente, nos llevó a plantear un diseño de enseñanza de las propiedades globales de las funciones estructurado y orientado por dichos estadios de desarrollo cognitivo, con el fin de favorecer el aprendizaje de los alumnos.

Posteriormente, Socas (2007) amplía el marco anterior desde la perspectiva del enfoque lógico semiótico (ELOS), enfoque que caracteriza mediante la elaboración de modelos de competencia teóricos y prácticos. Nuestro interés se centra en el Modelo de Competencia Cognitivo, que describe la comprensión de los conceptos desde el punto de vista de las relaciones entre los elementos del modelo: los sistemas de representación semióticos, los estadios de desarrollo cognitivo de los sistemas de representación, y las dificultades y errores en el aprendizaje. En este marco, el autor caracteriza los estadios utilizando acciones de aprendizaje (reconocimientos, transformaciones, conversiones y coordinaciones), con las que se puede describir el proceso de aprendizaje significativo del concepto a través de sus representaciones.

- Semiótico: Se reconocen los elementos de un SRS en relación con el objeto matemático.

- Estructural: Se realizan transformaciones en el anterior SRS y conversiones entre SRS, en las que hay un sistema que el alumno controla y facilita la conversión al otro.

- Autónomo: Se dominan diversos SRS para significar el objeto (al menos dos), y se coordinan de forma espontánea.

Siguiendo este modelo, que tiene como referencia el modelo de coordinación de registros de Duval (1993), consideramos que la adquisición de los conceptos matemáticos se alcanzará en el momento que haya una coordinación, libre de contradicciones, entre las diferentes representaciones del objeto matemático en cuestión.

La concreción del diseño de enseñanza, por una parte, necesita del estudio exhaustivo de los conceptos, para lo cual llevamos a cabo un análisis curricular de los mismos en la etapa de Educación Secundaria Obligatoria, y, por otra, debemos conocer cuáles son los errores y las dificultades que se manifiestan en su aprendizaje, con el fin de plantear estrategias de enseñanza orientadas a minimizar o evitar estos errores y dificultades de aprendizaje. Esta información la recogemos a lo largo del desarrollo de la investigación mediante el análisis de los datos del proceso de enseñanza-aprendizaje de los conceptos.

\section{METODOLOGÍA DEL DESARROLLO EXPE- RIMENTAL}

Dado el carácter de nuestra investigación, que nace en el aula al observar una situación educativa susceptible de mejora, hemos utilizado la metodología cualitativa de investigación acción para su desarrollo experimental, porque permite comprender y mejorar la propia práctica educativa (Kemmis y McTaggart, 1988), aunque también hemos hecho algunos cálculos estadísticos puntuales. 
Los estudios de Kemmis y McTaggar (1988), Elliot (1990), Pérez (1994) y Hopkins (1989) nos han permitido valorar esta metodología de forma positiva y comprobar que nuestra investigación cumple las especificaciones de la misma. En concreto, Pérez (1994, pág. 158-166) enuncia ciertos rasgos que caracterizan la investigaciónacción, que son propios de nuestra investigación y que describimos a continuación.

La investigación surge en el aula al observar un problema de enseñanza-aprendizaje de unos conceptos matemáticos, y se desarrolla desde una perspectiva ecológica, es decir, se integra docencia e investigación de manera que no se producen rupturas en el proceso educativo del grupo de alumnos donde se desarrolla la investigación, pero, en todo momento, los resultados de la investigación orientan la práctica educativa.

Este marco metodológico integra teoría y praxis. Este carácter nos permite integrar un diseño de enseñanza fundamentado en un marco teórico con la docencia que se deriva del mismo, una docencia que parte del conocimiento de los alumnos, está estructurada por estadios de desarrollo y actúa sobre las diversas representaciones de los conceptos.

Se han considerado diversas fuentes de información (departamento de matemáticas, profesor primer ciclo ESO, profesor observador, profesores secundaria, alumnos) y diversas herramientas para la recogida de la información (actas, entrevistas, cuestionarios, cuaderno alumnos, informes del observador externo, grabaciones, notas de campo). Esta diversidad de herramientas y fuentes de información ha contribuido a la validación del desarrollo y los datos obtenidos en la investigación, completando la información que aportaban los alumnos y la profesora investigadora en su actividad profesional. Por otra parte, toda la información obtenida ha sido tenida en cuenta de forma reflexiva, por la profesora-investigadora y por el director de la investigación para sentar las bases de la siguiente planificación o de las conclusiones de la investigación.

Hemos seguido una espiral de tres ciclos de investigación-acción, completando en cada uno de ellos sus fases características: planificación, acción, análisis y reflexión. En esta espiral, cada ciclo complementa ciertos aspectos del anterior; de hecho, las fases de cada ciclo se integran de forma dinámica y, con el fin de obtener nuevos datos que permitan obtener unas reflexiones más profundas, las reflexiones de un ciclo orientan la planificación del siguiente. Por otra parte, durante toda la investigación, se ha ido redactando un cuaderno de trabajo en el que se han reflejado multitud de datos, diario que ha constituido otro referente más para encaminar las acciones a seguir en el desarrollo experimental de la investigación.

\section{ANÁLISIS CURRICULAR}

Con el fin de observar el tratamiento didáctico de las propiedades globales de las funciones y de los contenidos sobre los que se fundamenta su aprendizaje en segundo ciclo de ESO, se ha realizado un análisis curricular de la unidad didáctica
«Funciones y Gráficas» en los ciclos primero y segundo de ESO. Este análisis curricular se llevó a cabo mediante la revisión de documentos oficiales (LOGSE, LOE, Decretos de Mínimos y Desarrollos Curriculares de la Junta de Castilla y León) y de los libros de texto de diversas editoriales: Anaya, SM, Santillana, Edelvives y Almadraba.

Aunque el diseño de enseñanza que elaboramos en la investigación va dirigido a plantear una docencia de las propiedades globales de las funciones en el segundo ciclo de ESO, realizamos el análisis curricular en toda la etapa educativa porque el aprendizaje de unos contenidos, propios del segundo ciclo, se fundamenta en el conocimiento de otros, que son propios del primer ciclo y, en el modelo de docencia que seguimos, que está inspirado en el marco ELOS, el aprendizaje de los símbolos nuevos se fundamenta en el conocimiento de los símbolos antiguos.

Este estudio permite conocer el tratamiento didáctico que hacen las diversas fuentes consultadas de las propiedades globales de las funciones, es decir, qué representación se utiliza para introducir el concepto, qué orientaciones proponen para que el alumno llegue a reconocer el concepto en los diversos sistemas de representación, y cómo plantean las traducciones entre las distintas representaciones. Por otra parte, este estudio también delimita de forma teórica los contenidos de esta unidad didáctica que corresponden a cada curso y la orientación didáctica correspondiente.

El análisis curricular muestra que las propiedades globales de las funciones se tratan en los cuatro sistemas de representación (verbal, gráfico, numérico y algebraico), asociados a funciones concretas, salvo en el caso de su definición formal-algebraica, en la que el concepto de función es genérico y está representado mediante el simbolismo funcional de las ordenadas.

Las diferencias de un texto a otro aparecen en el tratamiento didáctico de los conceptos, pues varía la representación que se utiliza para introducirlos y, por tanto, también la forma en que se plantea su desarrollo cognitivo a través de los sistemas de representación. En general, unas veces, se utiliza la definición verbal y/o algebraica para introducir el concepto y, a continuación, se hace su interpretación gráfica. Y, otras veces, la interpretación de la gráfica de una función, normalmente contextualizada, permite introducir la definición verbal o formal-algebraica del concepto. Se puede llegar a observar cierto grado de formalización pero, en general, no siempre se propone el reconocimiento de los conceptos en todas las representaciones, sobre todo en la representación numérica.

Destacamos que no siempre se relacionan los símbolos de las diferentes representaciones con el fin de facilitar el paso de una representación a otra. En concreto, es escasa la orientación sobre la formación de los intervalos numéricos que ubican la propiedad (monotonía, signo, etc.) en el sistema de referencia cartesiano, y no siempre se utiliza simbolismo funcional sobre las gráficas de las funciones o relacionado con expresiones verbales, que oriente la adquisición de la definición formal-algebraica de los conceptos y que ayude a conocer el significado de los símbolos que aparecen en esta expresión. 


\section{DISEÑO DE ENSEÑANZA}

El marco teórico y los antecedentes de la investigación orientan un diseño de enseñanza de las propiedades globales de las funciones en $4^{\circ}$. de ESO Opción B. Este diseño pretende facilitar el aprendizaje significativo de estos conceptos y, para ello, tiene en cuenta el modelo de aprendizaje que proporciona el marco teórico ELOS. Por tanto, hemos planteado la enseñanza de cada concepto a partir del conocimiento de los alumnos, y la hemos organizado por estadios de desarrollo cognitivo (semiótico, estructural y autónomo).

La caracterización que ofrece el marco ELOS de dichos estadios, junto con el análisis curricular, que nos permite observar el tratamiento didáctico que se hace de estos conceptos, nos llevan a concretar unos aprendizajes asociados a cada estadio de desarrollo. La determinación de estos aprendizajes está motivada, por una parte, por las representaciones de los conceptos, es decir, por el grado de abstracción de los símbolos asociados a cada una de ellas y la dificultad para pasar de una representación a otra, y, por otra parte, por el conocimiento de los alumnos antes de dicha docencia y por el nivel educativo en el que se desarrolla la docencia, ya que, por ejemplo, no se puede alcanzar la misma autonomía en el caso del dominio que en caso de la tendencia, porque en este nivel no se trabaja el concepto de límite.

En concreto, algunos aspectos que describen globalmente el diseño de enseñanza de los conceptos serían los siguientes:

- Fundamenta el aprendizaje de los símbolos nuevos en el significado de los símbolos antiguos, es decir, en los símbolos que constituyen el conocimiento de los alumnos.

- Está estructurado por los estadios de desarrollo que caracteriza el marco teórico ELOS, y actúa sobre las diversas representaciones -verbal $(\mathrm{V})$, gráfica $(\mathrm{G})$, numérica (N) y algebraica (A)-, partiendo de las representaciones más cercanas al sistema antiguo hasta alcanzar aquellas en las que la abstracción de sus símbolos es mayor.

- Unas acciones sobre las representaciones del concepto concretan el aprendizaje que se alcanza en cada estadio de desarrollo -reconocimientos $(\mathrm{R})$, transformaciones $(\mathrm{T})$, conversiones $\left(\mathrm{C}_{\rightarrow}\right)$ y coordinaciones $\left(\mathrm{C}_{\leftrightarrow}\right)-$.

- Se motiva una docencia orientada por las relaciones entre los símbolos, con el fin de evitar aprendizajes mecánicos y memorísticos. En concreto, se promueve el aprendizaje significativo por conversiones y coordinaciones entre las diferentes representaciones.

- Tiene en cuenta los errores y las dificultades de aprendizaje observadas en los ciclos de investigación, en el sentido de plantear estrategias de enseñanza que los aborden en el propio diseño.

La figura 1 representa de forma general la evolución del aprendizaje de los conceptos que nos ocupan por estadios y a través de sus representaciones

Se inicia el aprendizaje en el estadio semiótico, donde se alcanza el reconocimiento del concepto en el sistema de representación gráfico a partir del significado de los símbolos del sistema antiguo (conocimiento del alumno).

Figura 1

Esquema general de la evolución del aprendizaje de los conceptos por estadios (semiótico, estructural y autónomo) y a través de sus representaciones.

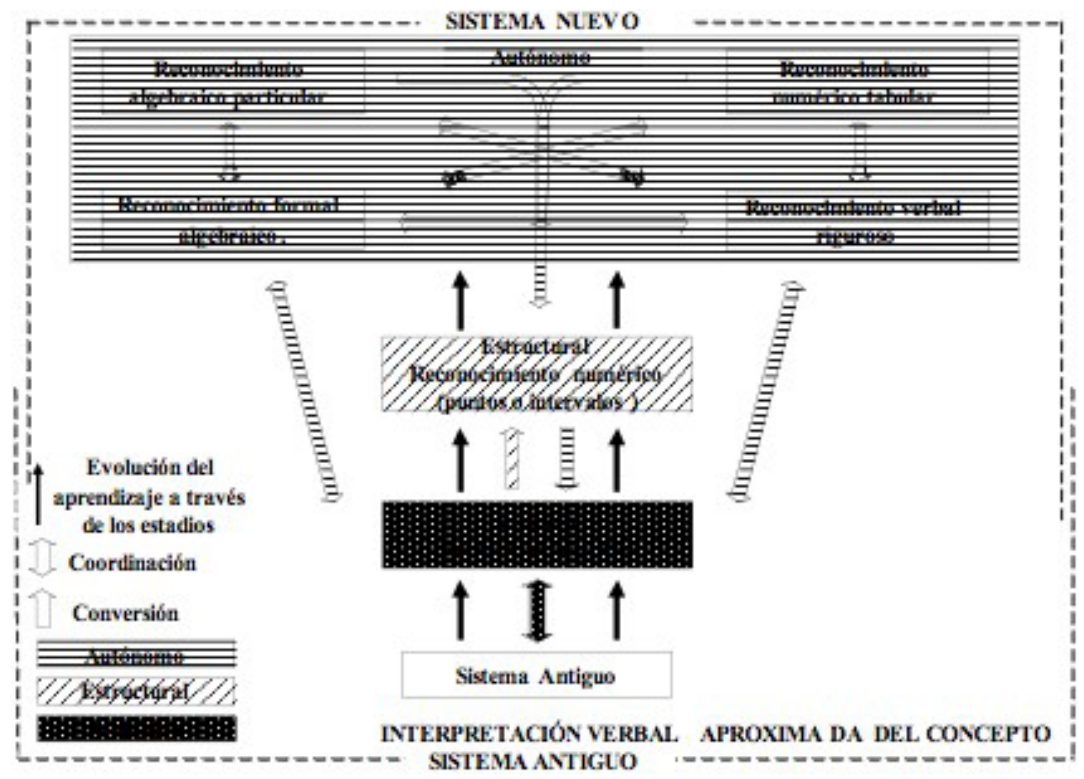


En el estadio estructural, se producen transformaciones sobre la representación gráfica del concepto que facilitan la conversión al sistema de representación numérico, donde se llega a reconocer el concepto. La conversión recíproca (numérica $\rightarrow$ gráfica) es menos intuitiva, porque la representación numérica aporta poca información sobre el carácter covariacional de los conceptos, por lo que la coordinación entre ambas representaciones se alcanza en el estadio autónomo. Además, la representación numérica no permite acceder a otras representaciones de estos conceptos debido a esta pérdida de información.

Por último, en el estadio autónomo, se alcanza el reconocimiento formal, por conversión desde la representación gráfica, y los alumnos pueden llegar a coordinar ambas representaciones. Para algunos conceptos, su reconocimiento formal permite reconocerlos en otros sistemas de representación, como el algebraico particular o un reconocimiento numérico tabular $\mathrm{y}$, aunque no se alcanza la correspondiente conversión recíproca, la representación de llegada refuerza el carácter del concepto en la representación de partida, la formal (trazo discontinuo).

Las diversas representaciones que sucesivamente se van adquiriendo a lo largo de todo el proceso de aprendizaje, y sobre todo en el estadio autónomo, en algunos casos, permiten independizar el concepto del soporte gráfico a partir del cual se ha iniciado su aprendizaje, y facilitan la adquisición del nuevo símbolo con significado propio, independiente de sus representaciones.

Es preciso resaltar que todas las acciones que tienen lugar en el aprendizaje del concepto se refuerzan y complementan durante la docencia mediante expresiones verbales aproximadas, descriptivas, que constituyen un modus relacional y, en suma, facilitan la adquisición y dominio de estas acciones por parte del alumno.

A título de ejemplo, presentamos el diseño de enseñanza del concepto de monotonía, indicando las acciones de aprendizaje que tienen lugar en cada estadio de desarrollo y ejemplificando algunas de estas acciones.

\section{Monotonía: crecimiento y decrecimiento}

Este contenido es muy intuitivo porque está asociado a muchos fenómenos de la vida cotidiana, por tanto, pertenece al conocimiento temprano del individuo. Sin embargo, la introducción del formalismo puede resultar complicada.

\section{Sistema antiguo}

Los símbolos del sistema antiguo que pudieran fundamentar directamente el aprendizaje del nuevo símbolo pudieran ser:

- Sistema de referencia: ejes cartesianos y coordenadas de un punto.

- Lectura e interpretación de gráficas de funciones dentro de un contexto.
- Concepto de función como dependencia entre variables. Interpretación gráfica.

- Sistemas de representación de funciones y traducciones más significativas.

- Se conoce la notación punto relleno-punto vacío del lenguaje gráfico y su correspondencia con la notación de intervalos de la recta real.

\section{Estadio semiótico}

Se distinguen y señalan sobre la gráfica los tramos donde la función es creciente, decreciente y constante. Las acciones de aprendizaje que estarían asociadas a este estadio serían: $\mathrm{R}_{\mathrm{V}(\mathrm{Apr})}, \mathrm{C}_{\mathrm{V}(\mathrm{Apr}) \rightarrow \mathrm{G}}, \mathrm{R}_{\mathrm{G}}, \mathrm{C}_{\mathrm{G} \rightarrow \mathrm{V}(\mathrm{Apr})}, \mathrm{C}_{\mathrm{G}} \leftrightarrow \mathrm{V}(\mathrm{Apr})$, cuyo significado presentamos a continuación:

- $\mathrm{R}_{\mathrm{V} \text {.(Aproximado) }}$ Un enunciado verbal aproximado permite interpretar el concepto en la gráfica de la función. Este enunciado utiliza símbolos del sistema antiguo y se expresa de forma que resulte cercano a la intuición del alumno.

$-\mathrm{C}_{\mathrm{V}(\mathrm{Apr}) \rightarrow \mathrm{G}}$ : El reconocimiento verbal aproximado de los conceptos permite interpretarlos en la gráfica de la función.

$-\mathrm{R}_{\mathrm{G}}$ : Se reconoce el concepto en el sistema de representación gráfico y se interpretan las variaciones del mismo en este sistema de representación.

$-\mathrm{C}_{\mathrm{G} \rightarrow \mathrm{V}(\mathrm{Apr})}$ : Se realizan conversiones del sistema de representación gráfico al verbal aproximado. La interpretación gráfica del concepto y sus variaciones se expresa de forma verbal aproximada.

$-\mathrm{C}_{\mathrm{G}} \leftrightarrow \mathrm{V(Apr)}$ : La interpretación verbal aproximada del concepto permite interpretarlo gráficamente y, recíprocamente, la interpretación gráfica del concepto se expresa verbalmente de forma aproximada.

La figura 2 ejemplifica la acción de reconocimiento gráfico:

Figura 2

Acción de aprendizaje.

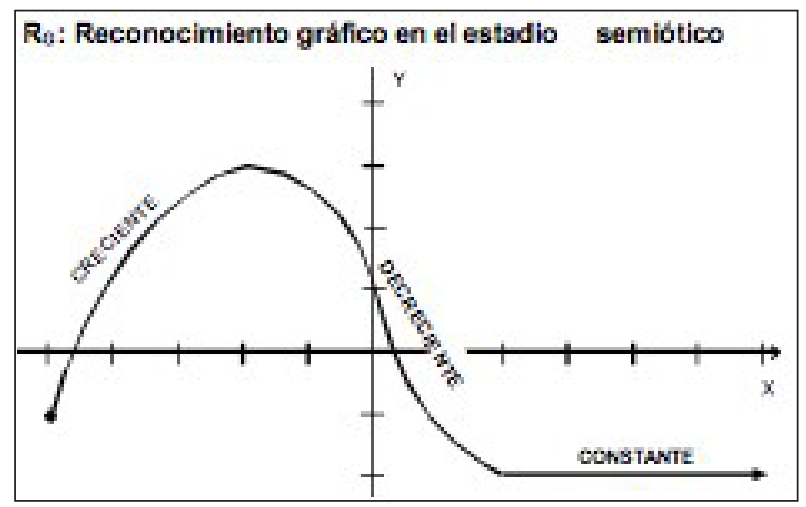




\section{Estadio estructural}

Se obtienen intervalos gráficos sobre el eje de abscisas como resultado de la proyección de la gráfica sobre dicho eje, en función de las variaciones de la monotonía. A continuación, se expresa dicha información mediante notación numérica de intervalos referida al eje de abscisas. Las acciones de aprendizaje ligadas a este estadio son: $\mathrm{T}_{\mathrm{G}}, \mathrm{C}_{\mathrm{G}} \rightarrow_{\mathrm{N}(\mathrm{I})}, \mathrm{R}_{\mathrm{N}(\mathrm{I})}$, y su significado:

$-\mathrm{T}_{\mathrm{G}}$ : Se realizan transformaciones internas en el sistema de representación gráfico. Se proyecta la gráfica en el eje de abscisas, en función del concepto o de las variaciones del concepto, obteniendo intervalos gráficos que ubican el concepto en el sistema de referencia cartesiano.

$-\mathrm{C}_{\mathrm{G} \rightarrow \mathrm{N}(\mathrm{I})}$ : Se realizan conversiones del sistema de representación gráfico al numérico. Se determinan los intervalos numéricos a partir de los intervalos gráficos obtenidos de la proyección de la gráfica en el eje de abscisas.

$-\mathrm{R}_{\mathrm{N}(\mathrm{I})}$ : Se interpreta el concepto mediante intervalos numéricos de la recta real.

La figura 3 ejemplifica la acción de la transformación gráfica.

Figura 3

Acción de aprendizaje.

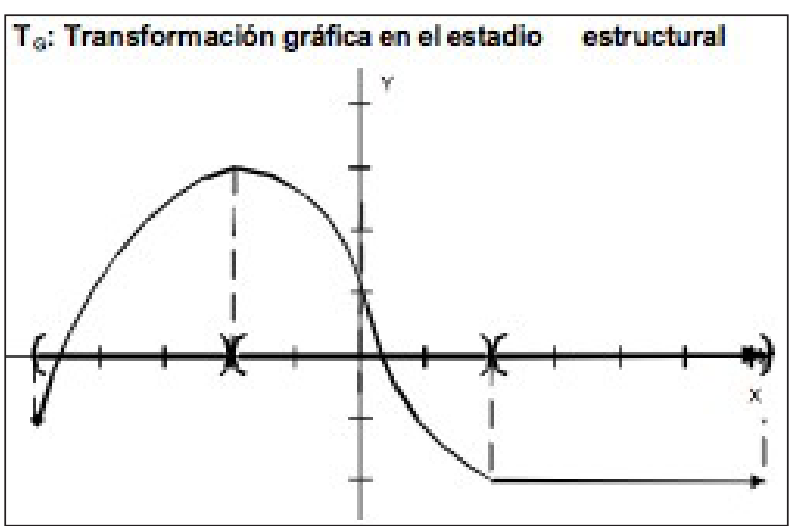

\section{Estadio autónomo}

Se plantea llegar a coordinar las representaciones gráfica y numérica, y gráfica y formal, ya sea a través de una expresión algebraica universal o una definición verbal rigurosa, por lo que también se coordinan estas dos últimas representaciones.

«Una función es creciente en un intervalo cuando al aumentar la variable independiente aumenta la variable dependiente en todo el intervalo».

$$
\text { Creciente: } x_{1}<x_{2} \Rightarrow f\left(x_{1}\right)<f\left(x_{2}\right) \quad\left(y_{1}<y_{2}\right)
$$

«Una función es decreciente en un intervalo cuando al aumentar la variable independiente disminuye la variable dependiente en todo el intervalo».

$$
\text { Decreciente: } x_{1}<x_{2} \Rightarrow f\left(x_{1}\right)>f\left(x_{2}\right) \quad\left(y_{1}>y_{2}\right)
$$

«Una función es constante en un intervalo cuando al aumentar la variable independiente no aumenta ni disminuye la variable dependiente, mantiene su valor constante en todo el intervalo».

$$
\text { Constante: } x_{1}<x_{2} \Rightarrow f\left(x_{1}\right)=f\left(x_{2}\right)\left(y_{1}=y_{2}\right)
$$

Para este concepto, su reconocimiento formal permite interpretarlo en el sistema de representación numérico tabular de las funciones: con una distribución creciente de las abscisas, se observa el comportamiento de las ordenadas.

Las acciones de aprendizaje propias de este estadio son las siguientes: $\mathrm{C}_{\mathrm{N}(\mathrm{I}) \rightarrow \mathrm{G}}, \mathrm{C}_{\mathrm{G} \leftrightarrow \mathrm{N}(\mathrm{I})}, \mathrm{C}_{\mathrm{G}} \rightarrow \mathrm{V}(\mathrm{Rig}), \mathrm{R}_{\mathrm{V}(\mathrm{Rig})}, \mathrm{C}_{\mathrm{G}} \rightarrow \mathrm{A}($ Uni $)$, $\mathrm{R}_{\mathrm{A}(\text { Uni })}, \quad \mathrm{C}_{\mathrm{V}(\mathrm{Rig}) \rightarrow \mathrm{G}}, \quad \mathrm{C}_{\mathrm{G}} \leftrightarrow \mathrm{V}$ (Rig) $, \quad \mathrm{C}_{\mathrm{A}(\text { Uni }) \rightarrow}, \mathrm{C}_{\mathrm{G}}, \mathrm{C}_{\mathrm{G}} \leftrightarrow \mathrm{A}_{\mathrm{A}(\text { Uni })}$,

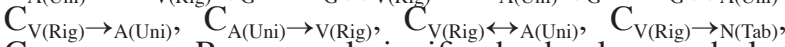
$\mathrm{C}_{\mathrm{A}(\text { Uni }) \rightarrow \mathrm{N}(\mathrm{Tab})}, \mathrm{R}_{\mathrm{N}(\mathrm{Tab})}$, y el significado de algunas de las cuales presentamos a continuación:

$-\mathrm{C}_{\mathrm{N}(\mathrm{I}) \rightarrow \mathrm{G}}$ : Se dibuja la gráfica de una función sujeta a unas condiciones de ubicación del concepto o de sus variaciones, dadas por unos intervalos numéricos, aunque, primero, se interpretan estos valores de forma gráfica en el eje de abscisas.

$-\mathrm{C}_{\mathrm{G} \rightarrow \mathrm{V}(\mathrm{Rig})}$ : Se realizan conversiones del sistema de representación gráfico al verbal. A partir de la representación gráfica del concepto se establece una expresión verbal que lo define con rigor.

$-\mathrm{R}_{\mathrm{V} \text { (Riguroso) }}$ : Una expresión verbal define el concepto de forma rigurosa.

$-\mathrm{C}_{\mathrm{V}(\mathrm{Rig}) \rightarrow \mathrm{G}}$ : La definición verbal del concepto permite interpretarlo sobre la gráfica de la función.

$-\mathrm{C}_{\mathrm{G}} \rightarrow_{\mathrm{A}(\text { Uni) }}$ : La representación gráfica del concepto permite enunciar una expresión formal algebraica que lo define.

$-\mathrm{R}_{\mathrm{A} \text { (Universal) }}$ : Se reconoce el concepto formalmente a través de una expresión algebraica universal que lo define.

$-\mathrm{C}_{\mathrm{A}(\text { Uni) } \rightarrow} \rightarrow_{\mathrm{G}}$ : La definición formal algebraica del concepto permite interpretarle sobre la gráfica de la función.

$-\mathrm{C}_{\mathrm{V}(\mathrm{Rig}) \rightarrow \mathrm{A}(\mathrm{Unni})}$ : Se realizan conversiones del sistema de representación verbal al algebraico. La definición verbal del concepto se expresa mediante una expresión algebraica formal.

$-\mathrm{C}_{\mathrm{A}(\text { Uni }) \rightarrow \mathrm{V}(\mathrm{Rig})}$ : La definición formal del concepto a través de una expresión algebraica se expresa verbalmente.

$-\mathrm{C}_{\mathrm{A}(\text { Uni) }} \rightarrow_{\mathrm{N}(\mathrm{Tab})}$ : La definición formal algebraica del 
concepto permite interpretarlo en la representación tabular de las funciones.

$-\mathrm{C}_{\mathrm{V}(\mathrm{Rig}) \rightarrow \mathrm{N}(\mathrm{Tab})}$ : Se realizan conversiones del sistema de representación verbal al numérico tabular. La definición verbal de un concepto permite interpretarlo en la representación tabular de las funciones.

$-\mathrm{R}_{\mathrm{N} \text { (Tabular) }}$ : Se reconoce el concepto en la representación numérica-tabular de las funciones y se interpretan las variaciones del mismo en este sistema de representación.

La doble conversión entre algunas de las representaciones lleva a la coordinación de las mismas, que es la propuesta que se hace para este estudio: coordinar las representaciones gráfica y numérica, y gráfica y formal (algebraica universal o definición verbal rigurosa).

La figura 4 ejemplifica la acción de conversión del sistema de representación gráfico al formal, ya sea a través de una expresión algebraica universal o a través de una definición verbal rigurosa. Se observa cómo sobre la gráfica se va introduciendo cierto simbolismo que facilita esta conversión.

Figura 4

Acción de aprendizaje.

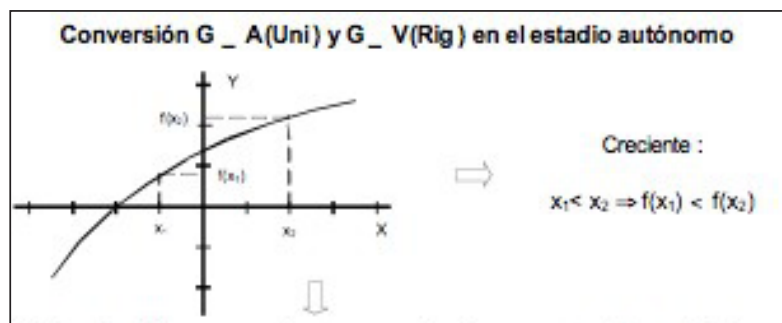

"Una función es creciente cuando al aumentar la variable indebendiente aumenta la variable dependiente"

La docencia que se deriva de este diseño se ha desarrollado en dos IES de Valladolid y provincia (Santa Teresa y Pío del Río Hortera), siguiendo una metodología de aula interactiva entre el profesor y los alumnos, aunque es el profesor quien introduce los conceptos en un primer momento.

\section{CONCLUSIONES}

Nuestra investigación utiliza la representación gráfica como medio para generar la visualización inicial de los conceptos, porque consideramos que esta representación facilita su interpretación inicial al aportar ciertas intuiciones sobre los mismos, y permite su desarrollo cognitivo a través de los diferentes sistemas de representación, por conversiones y coordinaciones, como se ha observado en el diseño de enseñanza. Además, las indicaciones del currículo oficial y las ideas de diversos autores en investigación educativa refuerzan este planteamiento. Así, Deulofeu (1995) y Bloch (2003) indican que la representación gráfica facilita el aprendizaje de conceptos asociados a ésta, al propiciar ciertas intuiciones sobre los mismos. Castro y Castro (1997) consideran que la información visual permite la formación de conceptos y el desarrollo de procedimientos, y refieren los estudios de diversos autores que confirman que el incremento en la capacidad de visualización producido por el uso de las representaciones gráficas ayuda a la comprensión de los conceptos matemáticos.

Por otra parte, Socas (2007) indica que el dominio del sistema de representación formal es más un objetivo del proceso de aprendizaje que un medio para el aprendizaje, y, en nuestro caso, la práctica educativa diseñada propicia que la definición formal de los conceptos se alcance en el proceso de aprendizaje de los mismos, es decir, a lo largo de su desarrollo cognitivo a través de los sistemas de representación. Por tanto, esta definición llega a formar parte de la experiencia del alumno y de sus esquemas conceptuales, coincidiendo con las ideas de Azcárate (1997).

El marco teórico ELOS estructura el proceso de aprendizaje de los conceptos por estadios de desarrollo: semiótico, estructural y autónomo. En este proceso, el conocimiento del alumno sienta las bases para introducir los nuevos símbolos, y la adquisición de las diferentes representaciones y las relaciones entre ellas va marcando la evolución entre los estadios. Esta interpretación del proceso de aprendizaje nos llevó a elaborar un diseño de enseñanza estructurado de acuerdo con la caracterización de dichos estadios y con las representaciones de los conceptos, con el fin de facilitar el aprendizaje de los alumnos. Partiendo de la representación gráfica, se alcanza el reconocimiento del concepto en otros sistemas de representación mediante transformaciones y conversiones, y la coordinación entre estas representaciones fundamenta el aprendizaje significativo de los conceptos y permite que todas las representaciones utilizadas den paso al objeto matemático.

Se observa que la adquisición de los conceptos es relativamente uniforme en los dos primeros estadios, es decir, el aprendizaje de todos ellos se produce en las representaciones gráfica y numérica. Sin embargo, éste aprendizaje está condicionado por la comprensión del concepto y por sus características de desarrollo cognitivo, lo que motiva que la evolución de un estadio al siguiente sea característica del concepto, aspectos que se han observado en el análisis del aprendizaje de los alumnos. En el estadio autónomo, debido a las limitaciones dadas por el nivel educativo en el que se desarrolla la docencia, no todos los conceptos se llegan a reconocer en todos los sistemas de representación, por lo que, el proceso de abstracción que se produce en el aprendizaje de cada concepto es distinto.

El carácter intuitivo del concepto dado por la cultura general no matemática, en general, ayuda a comprender el significado de estos conceptos matemáticos. En nuestro 
caso, en el estadio semiótico se evoluciona del carácter intuitivo que pueden tener los alumnos sobre el concepto hasta su reconocimiento significativo en un sistema de representación de notación rigurosa, el gráfico, el cual permite su desarrollo cognitivo. Sin embargo, a veces, este carácter puede dificultar el correcto y completo aprendizaje de los conceptos a través de las diversas representaciones. Además, el uso del lenguaje habitual para favorecer la interpretación de los signos matemáticos puede generar conflictos de precisión y confusiones semánticas (Socas, 1997, 2007). Así, por ejemplo, en el análisis de los aprendizajes de los alumnos, se observa que la periodicidad se asocia con la variable tiempo, que no se aceptan mínimos más altos que máximo, etcétera.

Se observa que el aprendizaje significativo de cada uno de los conceptos se fundamenta en la comprensión de la covariación o coordinación de lo que ocurre con las dos variables a la vez, ya descrita en Leinhardt et al. (1990) mediante la interpretación de situaciones contextualizadas lineales por alumnos de edades similares a las de los

\section{REFERENCIAS BIBLIOGRÁFICAS}

AZCÁRATE, C. (1995). Sistemas de representación. UNO Revista de Didáctica de las Matemáticas, 4, pp. 13-20.

AZCÁRATE, C. (1997). Si el eje de ordenadas es vertical, ¿qué podemos decir de las alturas de un triángulo? SUMA, 25, pp. 23-30.

BAGNI, G.T. (2004). Una experiencia didáctica sobre funciones en la escuela secundaria. RELIME Revista Latinoamericana de Investigación en Matemática Educativa, 7(1), pp. 5-23.

BLOCH, I. (2003). Teaching functions in a graphic milieu: what forms of knowledge enable students to conjecture and prove? Educational studies in mathematics, 52(1), pp. 3-28.

BOSCH, M., COMPTA, A., GASCÓN, J., URBANEJA, P.M.G. y LAMARCA, J.M. (1996). Matemáticas $2^{\circ}$. ciclo de ESO/ 1er curso. Almadraba. Barcelona.

BOSCH, M., COMPTA, A., GASCÓN, J., URBANEJA,

P.M.G. Y LAMARCA, J.M. (1997). Matemáticas $2^{\circ}$. ciclo de ESO/ $2^{\circ}$. curso (opción B). Almadraba. Barcelona.

CASTRO, E. y CASTRO, E. (1997). Representaciones y Modelización. En La educación matemática en la enseñanza alumnos con los que se ha experimentado. En cualquiera de las representaciones de los conceptos, una determinada relación de dependencia entre las dos variables caracteriza al objeto matemático, donde el cambio de una variable está relacionado con el cambio de la otra. Por tanto, todos estos conceptos tienen asociada la idea de dependencia funcional, y su aprendizaje contribuirá al desarrollo del razonamiento covariacional (Dolores y Cuevas 2007).

Finalmente, consideramos que este diseño de enseñanza, fundamentado en el marco teórico ELOS, permite observar una continuidad en el desarrollo cognitivo de los sistemas de representación de los conceptos, concretado en sucesivas acciones de aprendizaje, por lo que, proporciona continuidad al proceso de aprendizaje de los mismos, posibilitando que éste sea más significativo y evitando una memorización mecánica. Sin embargo, estas observaciones y otras se confirmarán cuando se analice la docencia que se deriva de este diseño y el proceso de aprendizaje de los alumnos que han posibilitado la experimentación de esta investigación, cuya descripción detallada abordaremos en otro artículo. secundaria. ICE de la Universidad Autónoma de Barcelona y Horsori. Barcelona, pp. 95-124.

COBO, P. (1995). Efectos de la utilización de gráficos en la traducción algebraica de problemas verbales. Estudio de caso de problemas verbales que combinan estructura semántica de cambio y de comparación. UNO Revista de Didáctica de las matemáticas, 4.

CÓLERA, J., GARCÍA, R., GAZTELU, I. y OLIVEIRA, M. J. (2002). Matemáticas $3^{\circ}$. Educación Secundaria. En tus manos. Anaya. Madrid.

CÓLERA, J., GARCÍA, R., GAZTELU, I., OLIVEIRA, M.J. y MARTÍNEZ, M.M. (2002). Matemáticas $4^{\circ}$. (Opción A) Educación Secundaria. En tus manos. Anaya. Madrid.

CÓLERA, J., GARCÍA, R., GAZTELU, I., OLIVEIRA, M.J. y MARTÍNEZ, M.M. (2002). Matemáticas $4^{\circ}$. (Opción B) Educación Secundaria. En tus manos. Anaya. Madrid.

CONSEJERÍA DE EDUCACIÓN JUNTA DE CASTILLA Y LEÓN (2002). Decreto 7/2002, de 10 de enero, por el que se establece el Currículo de la Educación Secundaria Obligatoria de la Comunidad de Castilla y León. BOCyL 16/01/2002. 
CONSEJERÍA DE EDUCACIÓN JUNTA DE CASTILLA Y LEÓN (2007). Decreto 52/2007, de 17 de mayo, por el que se establece el currículo de Educación Secundaria Obligatoria en la Comunidad de Castilla y León. BOCyL 23/05/2007.

DE LA HAZA, C., MARQUÉS, M. y NORTES, A. (2003). Matemáticas $4^{\circ}$. (opción B) Educación Secundaria. Santillana. Madrid.

DE LA HAZA, C., MARQUÉS, M. y NORTES, A. (2003). Matemáticas $4^{\circ}$. (opción A) Educación Secundaria. Santillana. Madrid.

DEULOFEU, J. (1995). Concepciones de los alumnos de secundaria sobre distintas gráficas de funciones. UNO Revista de Didáctica de las matemáticas, №. 4, pp. 6-16.

DOLORES, C. (2004). Acerca del análisis de funciones a través de sus gráficas: concepciones alternativas de estudiantes de bachillerato. Revista Latinoamericana de Investigación en Matemática Educativa, Vol. 7, Nº 3, pp.195-218.

DOLORES, C. y CUEVAS, I. (2007). Lectura e interpretación de gráficas socialmente compartidas. Revista Latinoamericana de Investigación en Matemática Educativa, 10(1), pp. 69-96.

DUVAL, R. (1993). Sémiosis et Noésis. Conférence A.P.M.E.P.I.R.E.M.

DUVAL, R. (1998). Registros de representación semiótica y funcionamiento cognitivo del pensamiento. Hitt, F. (ed.). Investigaciones en Matemática Educativa II, pp. 173-201. México: Departamento de Matemática Educativa del Cinvestav-IPN-Grupo Editorial Iberoamericana.

ELLIOT, J. (1990). La investigación-acción en educación. Morata. Madrid.

HOPKINS, D. (1989). Investigación en el aula. PPU, Barcelona.

KEMMIS, S. y MCTAGGART, R. (1988). Cómo planificar la investigación-acción. Laertes. Barcelona.

KNUTH, E. (2000): Student understanding of the Cartesian connection: an exploratory study. Journal for Research in Mathematics Education, 31(4), pp. 500-508.

LEINHARDT, G., ZASLAVSKY, O. y STEIN, M.K. (1990). Functions, graphs, and graphing: tasks, learning, and tea- ching. Review of Educational Research, 60(1), pp. 1-64.

MARTINEZ, J. (1969). Elementos de matemáticas. Tercera edición. Gráficas Ándrés Martín, S.A.

MEC (1990). Ley Orgánica 1/1990, de 3 de octubre, de Ordenación General del Sistema Educativo (LOGSE).

MEC (2006). Ley Orgánica 2/2006, de 3 de mayo, de Educación. BOE 04/05/2006.

MEC (2007). Real Decreto 1631/2006, de 29 de diciembre, por el que se establecen las enseñanzas mínimas correspondientes a la Educación Secundaria Obligatoria. BOE 05/01/2007.

PÉREZ, G. (1994). Investigación cualitativa. Retos e interrogantes. La Muralla. Madrid.

REDAL, E. J. y otros (2007). Matemáticas $3^{\circ}$. ESO. Proyecto La Casa del Saber. Santillana. Madrid.

SANZ LERMA, I. (2004). Expresiones gráficas del lenguaje matemático. UNO Revista de Didáctica de las matemáticas, 35, pp. 9-22.

SANZ REQUENA, J. F. (2002): Matemáticas ESO 3 er curso. Proyecto 2.2. Edelvives. Madrid.

SANZ REQUENA, J.F. (2003). Matemáticas ESO $4^{\circ}$. curso. Proyecto 2.2. Edelvives. Madrid.

SCHWARZ, B. y DREYFUS, T. (1995). New actions upon old objects: a new ontological perspective on functions. Educational Studies in Mathematics, 29, pp. 259-291.

SOCAS, M. (1997). Dificultades, obstáculos y errores en el aprendizaje de las matemáticas en la Educación Secundaria. En La educación matemática en la enseñanza secundaria. ICE de la Universidad Autónoma de Barcelona y Horsori. Barcelona, pp. $125-154$

SOCAS, M. (2007). Dificultades y errores en el aprendizaje del las matemáticas. Análisis desde el Enfoque Lógico Semiótico. En Investigación en Educación Matemática XI, pp.19.52.

VIZMANOS, J. R. y ANZOLA, M. (1998). Matemáticas. Algoritmo $20003^{\circ}$. Secundaria. S.M. Madrid.

VIZMANOS, J.R. y ANZOLA, M. (2003). Matemáticas $4^{\circ}$. Secundaria (opción B). S.M. Madrid. 


\section{Design of the teaching of global properties of functions through their graphs}

\author{
Ortega, Tomás ${ }^{1}$ y Pecharromán, Cristina ${ }^{2}$ \\ ${ }^{1}$ Didáctica de la Matemática. Universidad de Valladolid \\ ${ }^{2}$ IES Valverde de Lucerna. Puebla de Sanabria (Zamora) \\ ortega@am.uva.es \\ cpecharromang@yahoo.es
}

\begin{abstract}
Summary
This work focuses on the development of design education to facilitate and improve the teaching-learning process of the global properties of functions (domain, path, monotony, extreme, cutting with axes, sign, continuity, trend, periodicity, symmetry, convexity) in Second Cycle of Compulsory Secondary Education (students between 14 and 16 years old). We consider the learning of these concepts from a global point of view, without using the differential calculus, that is, the teaching process starts from a qualitative analysis of its graphical representation, and it continues till it reaches the other representations.
\end{abstract}

The previous investigations -Cobo (1995), Bagni (2004), Dolores (2004) and Dolores and Cuevas (2007), Deulofeu (1995) and Bloch (2003) - are a guide for learning global properties of functions from their graphical representation and visualization processes. On the other hand, Azcárate (1997), Knuth (2000), Bloch (2003), echo Schwarz and Dreyfus (1995) ideas, Duval (1993, 1998) and Bagni (2004), and they point at the relationship between representations of concepts. Finally, other researches -Leinhardt, Zaslavsky and Stein (1990), Deulofeu (1995), Dolores (2004), Dolores and Cuevas (2007) - guide us on the difficulties of learning these concepts.

The theoretical framework, known as Logical Approach Semiotic (ELOS) Socas (1997 and 2007), is based on the model of coordination of records Duval (1993). This framework guides a teaching design structured in stages of cognitive development (semiotic, structural and autonomous), and in it, learning the concepts is based on the coordination of at least two of their representations. Following this theoretical framework, the learning associated with each stage is specified by actions (awards, transformations, conversions and coordinations) related to various representations of the concept. Thus, the stages of cognitive development are interpreted both as a guide to the teaching process and as a way of structuring it, sequencing the presentation of content. However, what ultimately shapes the learning of mathematical concepts are the representations of each concept and their relations.
This research begins in the classroom and tries to get results that will guide the educational practice, which makes the qualitative methodology of action research and has been the most suitable for experimental development. Although we have also made some calculations from time to time, we have basically followed this methodology which has been described by Kemmis and McTaggar (1988), Hopkins (1989), Elliot (1990), and Perez (1994), among other authors. In this methodology, as in our research, each cycle is planned taking into account the reflection on the action and the analysis of the previous cycle, and the future development of the cycles has improved its practice.

In this article, on the one hand, we present a curricular analysis of concepts treated in order to observe the treatment of learning the global properties of functions and contents on which their learning is based in the Second Cycle of Compulsory Secondary Education. On the other hand, as an example of this application, we describe the design of teaching applied to the concept of monotony, pointing out accurately all the actions which correspond to each of the stages of cognitive development.

Finally, we present the conclusions of our research, among which we point out the utility of the design carried out, in which the design leads to express the continuity in the cognitive development of systems of representation of concepts in the learning process, and, also, because it leads to the detection of the intrinsic characteristics of the concepts treated, such as the influence of the intuitive nature of these concepts and the covariacional character which supports their learning. Finally, considering that the formal representation is more a goal of the learning process than a means, this design allows to corroborate the ideas of Azcárate (1997) and Socas (2007) in the sense that such representations become a part of the students' experience and their conceptual schemes.

Key words: design, teaching, properties, functions, ELOS. 
